\title{
A. Baaj, P.V. Mummaneni, J.S. Uribe, A.R. Vaccaro, M.S. Greenberg (eds): Handbook of spine surgery
}

\author{
Thieme, New York, 2016 (ISBN 9781626231634)
}

\author{
Ronald Bartels ${ }^{1}$
}

Received: 15 February 2016 / Accepted: 18 February 2016/Published online: 2 March 2016

(C) Springer-Verlag Wien 2016

The editors and contributors should be highly commended for this very valuable contribution in the literature of spinal pathology and its surgical treatment. The format of the book and also the content completely justify the designation as handbook. In a very concise way, the most relevant but also recently updated information is given about a very large amount of subjects. Although the focus is spine surgery, the merits of conservative treatment are also highlighted. The subjects that are dealt with cover many daily practices, varying from private to academic. The audience for this book will be broad: from residents in a field that is related to spinal pathology to full clinical professors. Although sometimes the management of a disease is considered from the point of view of someone working in the United States (e.g. Ch. 39, p. 277: "The current indications for cervical arthroplasty in the United States ...", without making a statement about other countries) and most of the contributors are from the United States, the knowledge can perfectly been used in Europe and, I assume, also other countries.
The organisation of the handbook will facilitate the reader. It is divided into four chapters: "Anatomy", "Clinical spine surgery", "Spinal pathology" and "Surgical techniques". Each chapter is divided by subheadings. Long paragraphs of text are not used. Instead, every item is addressed point by point. The chapters end with common clinical questions, which can be used as a rehearsal of what has been read. A section that should not be overlooked is "Appendices". Three are incorporated: "Positioning", "Selected spinal orthoses" and "Outcome scales". In the appendix, "Positioning", the most common positionings are addressed by highlighting very concisely and point by point the issues that should be taken care of. In the "Selected spinal orthoses" appendix, the benefits and limitations of common orthoses are summarised. Finally, in "Outcome scales", a few outcomes are discussed. These appendices will turn out to be helpful in daily practice.

In conclusion, I recommend this handbook to everyone who is involved in the treatment of patients with spinal pathology. It will turn out to be a very handsome and valuable reference work.

Ronald Bartels

Ronald.bartels@radboudumc.nl

1 Department of Neurosurgery, Radboud University Medical Centre, Nijmegen, The Netherlands 